OPEN ACCESS

Edited by:

Thimios Mitsiadis,

University of Zurich, Switzerland

Reviewed by:

Virginia Tirino,

Università della Campania Luigi

Vanvitelli, Italy

Sofia Avnet,

University of Bologna, Italy

${ }^{*}$ Correspondence:

Taeko Kobayash

kobayashi.taeko.7e@kyoto-u.ac.jp

Specialty section:

This article was submitted to

Stem Cell Research,

a section of the journal

Frontiers in Cell and Developmental

Biology

Received: 21 August 2021

Accepted: 22 October 2021

Published: 04 November 2021

Citation:

Zhang J, Uchiyama J, Imami K, Ishihama $Y$, Kageyama $R$ and Kobayashi T (2021) Novel Roles of

Small Extracellular Vesicles in

Regulating the Quiescence and

Proliferation of Neural Stem Cells.

Front. Cell Dev. Biol. 9:762293.

doi: 10.3389/fcell.2021.762293

\section{Novel Roles of Small Extracellular Vesicles in Regulating the Quiescence and Proliferation of Neural Stem Cells}

\author{
Jingtian Zhang ${ }^{1,2}$, Junki Uchiyama ${ }^{3}$, Koshi Imami $^{3,4}$, Yasushi Ishihama ${ }^{3}$, \\ Ryoichiro Kageyama $^{1,2,5}$ and Taeko Kobayashi ${ }^{1,2 *}$ \\ ${ }^{1}$ Graduate School of Biostudies, Kyoto University, Kyoto, Japan, ${ }^{2}$ Institute for Frontier Life and Medical Sciences, Kyoto
University, Kyoto, Japan, ${ }^{3}$ Graduate School of Pharmaceutical Sciences, Kyoto University, Kyoto, Japan, ${ }^{4}$ PRESTO, Japan
Science and Technology Agency (JST), Tokyo, Japan, ${ }^{5}$ RIKEN Center for Brain Science, Wako, Japan
}

Neural stem cell (NSC) quiescence plays pivotal roles in avoiding exhaustion of NSCs and securing sustainable neurogenesis in the adult brain. The maintenance of quiescence and transition between proliferation and quiescence are complex processes associated with multiple niche signals and environmental stimuli. Exosomes are small extracellular vesicles (sEVs) containing functional cargos such as proteins, microRNAs, and mRNAs. The role of sEVs in NSC quiescence has not been fully investigated. Here, we applied proteomics to analyze the protein cargos of sEVs derived from proliferating, quiescent, and reactivating NSCs. Our findings revealed fluctuation of expression levels and functional clusters of gene ontology annotations of differentially expressed proteins especially in protein translation and vesicular transport among three sources of exosomes. Moreover, the use of exosome inhibitors revealed exosome contribution to entrance into as well as maintenance of quiescence. Exosome inhibition delayed entrance into quiescence, induced quiescent NSCs to exit from the G0 phase of the cell cycle, and significantly upregulated protein translation in quiescent NSCs. Our results suggest that NSC exosomes are involved in attenuating protein synthesis and thereby regulating the quiescence of NSCs.

Keywords: exosomes, neural stem cells, proteomics, quiescence, proliferation, small extracellular vesicles, protein synthesis, ribosomes

\section{INTRODUCTION}

NSCs, which are derived from the neuroepithelium of the neural tube, maintain the ability to selfrenew and to give rise to neurons, astrocytes, and oligodendrocytes throughout a whole life. Two generally accepted canonical domains in the adult central nervous system that keep a reservoir of NSCs are the subventricular zone near the lateral ventricles and the subgranular zone of the dentate gyrus, where most of the NSCs remains quiescent (Cheung and Rando, 2013; Urban et al., 2019; Kobayashi and Kageyama, 2021). The quiescent state of NSCs, which is characterized by low metabolic rate and low protein and RNA synthesis, is vital to the preservation of their genomic integrity and essential functional properties (Cavallucci et al., 2016). Quiescent NSCs (qNSCs) rest at the G0 phase of the cell cycle and do not express proliferation markers, such as Ki-67 and MCM2 (Codega et al., 2014). However, they can be reactivated and re-enter the cell cycle upon proper signals. NSCs maintain a delicate balance with proliferating and quiescence. This equilibrium is essential for NSC homeostasis, and its disruption may lead to brain aging and its associated diseases (Cavallucci et al., 2016). Discrete NSC niche stimuli play indispensable roles in determining whether 
NSCs remain quiescent or reenter the cell cycle (Fuentealba et al., 2012; Kjell et al., 2020). Neurotrophin-3 is secreted by endothelial cells in the brain and choroid plexus and maintains NSC quiescence (Delgado et al., 2014), while Sphingosine-1phosphate (S1P) and prostaglandin-D2 (PGD2) are G-protein coupled receptors in the cerebrospinal fluid (CSF) and actively sustain NSC quiescence (Codega et al., 2014). Though the transition between proliferating NSCs (active NSCs: aNSC) and qNSCs has been intensively studied, its mechanism has not been fully elucidated.

Extracellular vesicles are heterogeneous cell-derived membrane components that can be roughly categorized into small extracellular vesicles (sEVs, exosomes) and microvesicles (Van Niel et al., 2018). sEVs are intraluminal vesicles (ILVs) formed by inward budding of the endosomal membrane during the maturation of multiple vesicle endosome (MVE), then are secreted outside of the cell by the fusion of plasma membrane and MVE. The sEVs vary widely in size but, like ILVs, are normally within the range of $30-100 \mathrm{~nm}$ and generally do not exceed $150 \mathrm{~nm}$. Microvesicles are formed by direct budding from the plasma membrane and range in size from 100 to $1,000 \mathrm{~nm}$ (Colombo et al., 2014). Growing evidence has demonstrated the important roles of $\mathrm{sEVs}$ in signaling and regulating target cells via their diverse cargos, including protein, miRNA, and mRNA and various functional units (Valadi et al., 2007). The expression pattern of integrins in sEVs from cancer tissue differs in various kinds of cancers, which would partially account for the distinct metastatic destinies of various malignancies (Dolo et al., 1998; Hoshino et al., 2015). Ample evidence has proved that exosomes participate in the cell cycle and influence cell proliferation. Cancer cell-secreted exosomes increased the G1phase cancer cell ratio and enhanced cell proliferation (Huang et al., 2019; Matsumoto et al., 2020). Exosomal miRNA, which is derived from the choroid plexus of the lateral ventricle, posttranscriptionally regulates quiescent NSC differentiation (Lepko et al., 2019). EVs secreted from NSCs have also been reported to be involved in Statl pathway in target cells and to act as a microglia morphogen (Cossetti et al., 2014; Morton et al., 2018). Moreover, EVs derived from differentiated neural stem/ progenitor cells (NSPCs) have been reported to trigger the differentiation of proliferating NSPC (Stronati et al., 2019).

Despite the extensive research on the functions of EVs in NSCs (Batiz et al., 2015), the roles of sEVs in regulating the transition between aNSCs and qNSCs have not been certified. In the present study, we employed proteomics approaches to identify the functional differences among sEVs secreted from aNSCs, qNSCs and reactivating NSCs (reNSCs) in vitro. Our results demonstrated that the sEVs derived from the three sources differed in terms of protein content. From proteomics results, we hypothesized that NSCs might discard some proteins, such as ribosomes, to control the quiescence. To test this hypothesis, we treated NSCs with an inhibitor of sEV secretion during quiescence induction and resting. The inhibitor treatments facilitated NSC alterations from quiescence to proliferation. The expression of cell proliferation markers, MCM2, Ki-67, and $\mathrm{CycD1}$, was upregulated in quiescent NSCs, as well as quiescence-induced NSCs, after inhibitor treatments. Exosome inhibition activated protein translation in quiescent and quiescent-induced NSCs, which might fit well with the "discarding model" in which some ribosomes are discarded to maintain low protein translation. Our findings confirmed that sEVs are involved in the maintenance of qNSCs, as well as their transition between proliferation and quiescence.

\section{METHODS}

\section{Cell Culture, Quiescence Induction, and Reactivation}

NSCs were derived and previously established from ICR mouse embryos at E14.5 (Kobayashi et al., 2019). NSCs were frozen several times and then passed about five times before use. NSCs were able to differentiate into neural and glial fates after the inductions (not shown). Active NSCs were cultured in proliferation medium $[20 \mathrm{ng} / \mathrm{ml}$ epidermal growth factor (EGF); R\&D systems, Inc., Minneapolis, MN, United States), 20 ng/ml bFGF (FUJIFILM Wako Pure Chemical Corporation, Osaka, Japan), penicillin/streptomycin (Nacalai Tesque, Kyoto, Japan), and N-2 Plus supplement (R\&D Systems) in DMEM/F-12 (Gibco, Grand Island, NY, United States)] with $2 \mu \mathrm{g} / \mathrm{ml}$ laminin (Sigma-Aldrich Corp., St. Louis, MO, United States). Quiescence was induced with quiescent medium [50 ng/ml BMP4 (R\&D Systems) in proliferation medium minus EGF] after two washings with phosphate-buffered saline (PBS; Nacalai Tesque) and achieved in $3 \mathrm{~d}$. To prepare reNSCs, qNSCs were washed twice with PBS and cultured in proliferating medium for $2 \mathrm{~d}$. For the exosome inhibition, aNSCs were cultured for $1 \mathrm{~d}$ and the medium was supplemented either with $10 \mu \mathrm{M}$ GW4869 (Cayman Chemical Co., Ann Arbor, MI, United States) or dimethyl sulfoxide (DMSO; Nacalai Tesque) as the control. To measure protein synthesis, O-propargyl-puromycin (OP-puro, OPP; MedChemExpress Co., Monmouth Junction, NJ, United States) (Liu et al., 2012) was administrated for $1 \mathrm{~h}$ with $50 \mu \mathrm{M}$ OP-puro in culture medium, followed by fixation with $4 \%$ paraformaldehyde (PFA; Nacalai Tesque) in PBS.

\section{Exosome Extraction}

Supernatants from $15 \mathrm{~cm}$ culture dishes (Greiner, Oberosterreich, Austria) were centrifuged at $2,000 \times \mathrm{g}$ at $4^{\circ} \mathrm{C}$ for $20 \mathrm{~min}$ and at $10,000 \times \mathrm{g}$ at $4^{\circ} \mathrm{C}$ for $20 \mathrm{~min}$ and passed through a $0.22 \mu \mathrm{m}$ filter (TPP, Trasadingen, Switzerland). The filtered supernatants were concentrated using a $100 \mathrm{kDa} \mathrm{MW}$ centrifugal filter (Amicon Ultra-15; EMD Millipore, Billerica, MA, United States) at 5,000 $\times$ $g$ and then ultracentrifuged in a Beckmann optima XE with a SW 41 rotor (Beckman Coulter, Brea, CA, United States) at 100,000 $\times$ $\mathrm{g}$ at $4^{\circ} \mathrm{C}$ for $1 \mathrm{~h}$. The pellets were washed with cold PBS and recentrifuged at $100,000 \times \mathrm{g}$ at $4^{\circ} \mathrm{C}$ for $1 \mathrm{~h}$. The exosome pellets were recovered overnight in $\mathrm{PBS}$ at $4^{\circ} \mathrm{C}$ with gentle shaking and subjected to silver staining, western blot, particle analyses, and mass spectrometry. A total exosome isolation kit (Thermo Fisher Scientific, Waltham, MA, United States) was also used to collect exosomes from culture medium after inhibitor treatments by following the manufacturer's protocol. The exosome pellets were recovered in PBS and subjected to NanoSight (Malvern 
Instruments, Malvern, United Kingdom) measurements. The exosome concentrations collected by the kit were $5.88 \mathrm{E} 8 \pm 6.06 \mathrm{E} 6, \quad 7.51 \mathrm{E} 8 \pm 2.51 \mathrm{E} 7$, and $7.28 \mathrm{E} 8 \pm 3.97 \mathrm{E} 7$ particles/ml from 2-d aNSC, 3-d qNSC, and 2-d reNSC cultures, respectively.

\section{Protein Quantification}

Exosome proteins were quantified with a Micro $\mathrm{BCA}^{\mathrm{TM}}$ protein assay kit (Thermo Fisher Scientific). Bovine serum albumin (BSA) standard solutions and exosome samples were diluted in lysis buffer [50 mM Tris- $\mathrm{HCl}(\mathrm{pH} 8.0), 100 \mathrm{mM}$ $\mathrm{NaCl}, 5 \mathrm{mM} \mathrm{MgCl}_{2}$, and $0.5 \%$ (w/v) Nonidet P-40] and subjected to the manufacturer's protocol. The protein concentrations in total cell lysates were quantified by the Lowry method using DC protein assay reagents (Bio Rad Laboratories, Hercules, CA, United States).

\section{Western Blot and Silver Staining}

The cells were washed with cold PBS, lysed with lysis buffer [50 mM Tris- $\mathrm{HCl}(\mathrm{pH} 8.0), 100 \mathrm{mM} \mathrm{NaCl}, 5 \mathrm{mM} \mathrm{MgCl}$, $0.5 \%(\mathrm{w} / \mathrm{v})$ Nonidet P-40, Complete ${ }^{\mathrm{TM}}$ protease inhibitor cocktail (Roche Diagnostics, Basel, Switzerland), $1 \mathrm{mM}$ phenylmethylsulfonyl fluoride, $250 \mathrm{U} / \mathrm{ml}$ Benzonase (Sigma), $\quad 10 \mathrm{mM} \quad \beta$-glycerophosphate, $1 \mathrm{mM}$ sodium orthovanadate, $1 \mathrm{mM} \mathrm{NaF}$, and $1 \mathrm{mM}$ sodium pyrophosphate] on ice for $30 \mathrm{~min}$, and subjected to SDSPAGE. The exosome samples were either directly mixed with SDS sample buffer or first precipitated using $10 \%(\mathrm{v} / \mathrm{v})$ trichloroacetic acid (TCA) and resolved in sample buffer. The following primary antibodies were used for WB: rabbit anti-actin (Sigma-Aldrich), rabbit anti-CD9 (BioVision, Inc., Milpitas, CA, United States), rat anti-MFG-E8 (R\&D Systems), mouse anti-CD63 (Novus Biologicals, Littleton, CO, United States), rabbit anti-Sox2 (EMD Millipore), and mouse anti-Cycd1 (Sigma-Aldrich). Horseradish peroxidase (HRP)-conjugated anti-rabbit, anti-mouse, and anti-rat (Thermo Fisher Scientific) were used as the secondary antibodies. Western blots (WBs) were visualized by chemiluminescence using Amersham ECL or ECL prime (Cytiva, Tokyo, Japan), quantified on an LAS3000 image analyzer (Fujifilm, Tokyo, Japan), and normalized against the corresponding intensity of $\beta$-actin for cell lysates. Statistical analyses were performed using GraphPad Prism 8 (GraphPad Software, La Jolla, CA, United States). For silver staining, a Pierce silver stain kit was used (Thermo Fisher Scientific).

\section{Cell Fixation and Staining}

Cells labeled with OP-puro were fixed with 4\% PFA-PBS and stained with Alexa488-azide using Click-iT chemistry (Thermo Fisher Scientific). For immunocytochemistry to detect Ki-67 and MCM2, 4\% PFA-fixed cells were immunostained with mouse anti-Ki-67 (BD Pharmingen, Franklin Lakes, NJ, USA) and rabbit anti-MCM2 (Abcam, Cambridge, United Kingdom) as the primary antibodies and then stained with anti-mouseAlexa488 and anti-rabbit-Alexa594 as the secondary antibodies (Thermo Fisher Scientific) respectively and co-stained with DAPI
(Sigma). Cell images were obtained on an AF6000 (Leica Microsystems $\mathrm{GmbH}$, Wetzlar, Germany) and analyzed using ImageJ software (NIH, Bethesda, Maryland, United States). Statistical analyses were performed using GraphPad Prism 8 (GraphPad Software).

\section{Exosome Particle Size Measurement}

Exosome particle size distribution and concentration were evaluated with NanoSight NS300 (Malvern Instruments). The size distribution was determined from five videos recorded by the NanoSight. For the exosome concentration determinations, 20 videos were recorded for more precise results (Parsons et al., 2017).

\section{Transmission Electron Microscopy}

The exosome samples were subjected to negative staining by being loaded on the collodion surface of mesh grid (Nisshin EM, Tokyo, Japan), coated with $2 \%(\mathrm{w} / \mathrm{v})$ uranyl acetate, and left to dry on the grid. They were then photographed under a JEM-1400 TEM (JEOL Ltd., Akishima-Shi, Japan).

\section{RESULTS}

\section{Purifications of Exosomes Secreted From aNSCs, qNSCs and reNSCs}

We investigated the roles of sEVs secreted from NSCs in quiescent and proliferating conditions using cultured NSCs. To purify sEVs from cultured NSCs with high quality, we selected ultracentrifugation as the optimal method to collect abundant and pure sEVs (Figure 1A). In our method, cell culture supernatants were centrifuged to remove cell debris and passed through a $0.22 \mu \mathrm{m}$ filter to remove larger vesicles since sEVs is usually no larger than $150 \mathrm{~nm}$ in size. Nanoparticle tracking analysis (NTA) with NanoSight revealed that the estimated diameter of the purified sEVs was in the range of 100-130 nm (Figure 1B). Transmission electron microscopy (TEM) revealed their donut-shaped morphology (Figure 1C). Results from both NTA and TEM analyses showed that the purified sEVs had typical exosomal characters. Previous reports used the term "exosome" to refer to a mixture of small vesicles because there was heretofore no method of fully separating them (Mathieu et al., 2019). In the present study, we use the term "exosome" to refer the sEVs. We then obtained exosomes from active, quiescent, and reactivating NSCs. BMP was used to induce quiescence in NSCs in vitro (Martynoga et al., 2013). Exosomes from aNSCs (aExo) were purified from aNSC culture supernatants after NSCs were cultured in proliferating media for $2 \mathrm{~d}$. Quiescence was induced by culturing NSCs in BMP-containing quiescent medium for $3 \mathrm{~d}$ and exosomes from qNSCs (qExo) were collected from the supernatants. qNSCs were reactivated by changing medium from quiescent to proliferating medium, and exosomes from reNSCs (reExo) were collected after the NSCs were cultured for $2 \mathrm{~d}$. WB showed that exosome markers (CD9, CD63, and MFG-E8) (Thery et al., 2006) were expressed in the purified exosomes (Figure 1D). The transcriptional factor Sox 2 was used as a negative control to 


\section{A

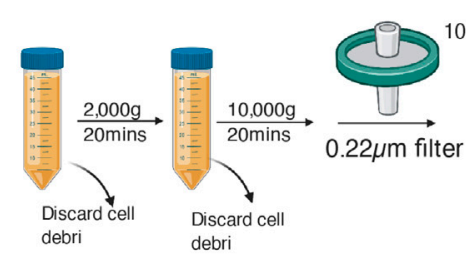

B
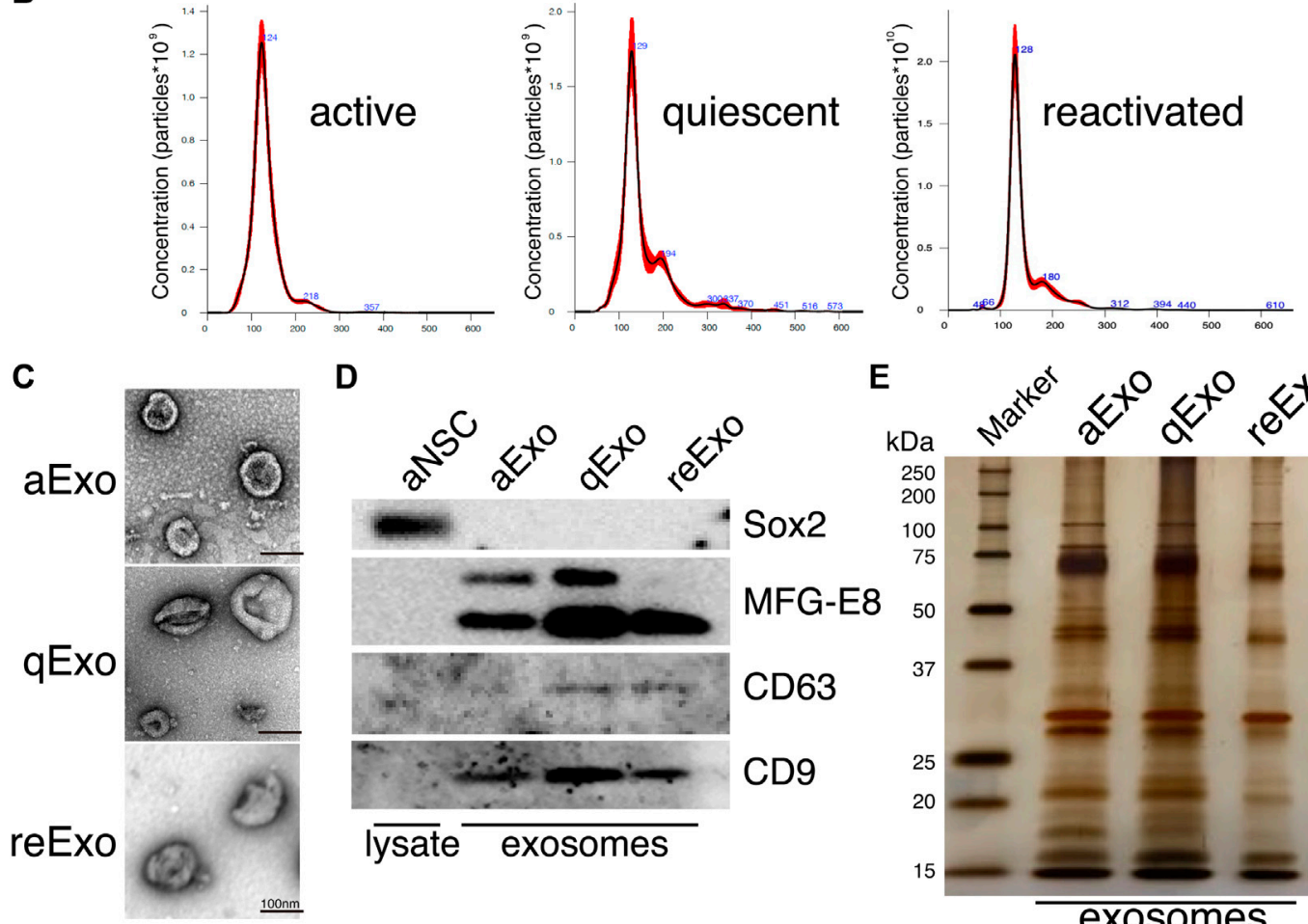

D
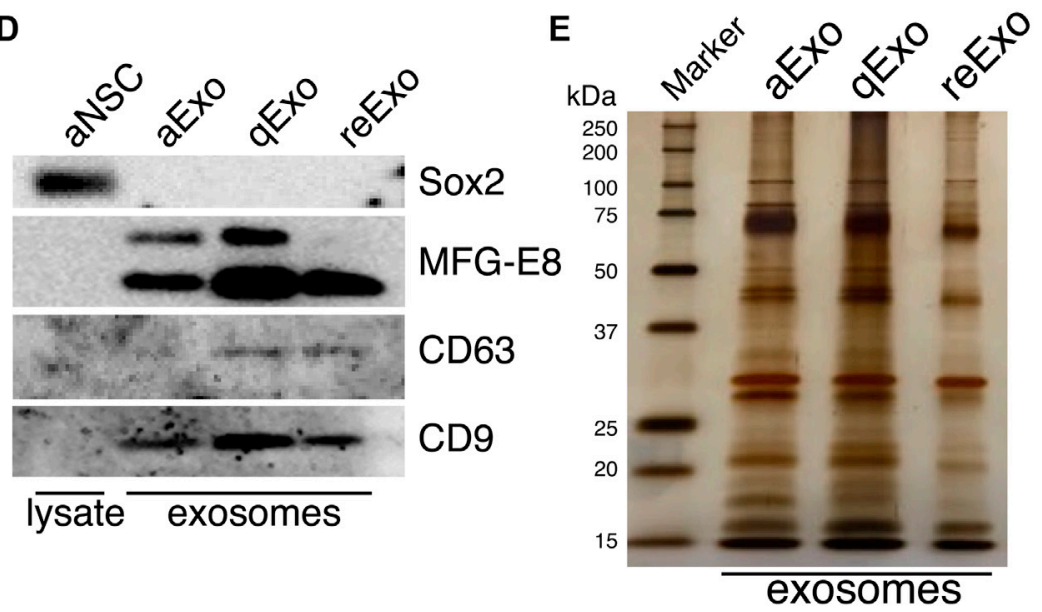

FIGURE 1 |NSC exosomes collection and quality examination. (A) Exosome collection workflow. (B) Exosome size distribution and concentration. Exosomes were prepared from media for 2, $3 \mathrm{~d}$, and 2 d incubation of active, quiescent, and reactivating NSCs, respectively. Averaged finite track length adjustment indicates exosome concentration and size, and was examined by NanoSight $(n=5)$. $X$-axis is vesicle size and $y$-axis is vesicle concentration. Numbers (blue) are approximate vesicles sizes at peak vesicle concentrations. (C) Transmission electron microscopy images of aExo, qExo, and reExo. Scale bar = $100 \mathrm{~nm}$. (D) Total cell lysates and exosomes were subjected to WB of Sox2, MFG-E8, CD9, and CD63. MFG-E8 showing two bands indicating two variants. (E) Silver staining of exosome samples (0.4 $\mu$ g). Left lane: molecular weight marker.

rule out possible cell debris contamination (Figure 1D). Taken together, the foregoing data suggest that we successfully purified exosome samples suitable for use in the subsequent assays and experiments.

\section{Proteomic Profiling of Exosomes Derived From NSCs}

We initially conducted silver staining to visualize proteomic inequalities and profiled the exosome protein contents. Silver staining showed similar protein expression patterns in the exosomes from active, quiescent, and reactivating NSCs (Figure 1E). We then exanimated the exosome profiles by nano-scale liquid chromatography/tandem mass spectrometry (nano LC/MS/MS) (Supplementary Methods). Digested proteins from individual exosome samples were labeled with nine different Tandem mass tag (TMT) 10-plex reagents (aExo vs. qExo vs. reExo; $n=3$ each) (Ogata and Ishihama, 2020). Relative protein abundance was quantified based on the "reporter ion intensity." Among 1,283 proteins we identified, 1,178 proteins were quantified in at least two out of the three replicates in at least one condition and were used for further analysis (Supplementary Table S1). To compare the exosome contents in the databases, we consulted the lists of proteins in Exocarta which includes those involved in MVB biogenesis, those associated with membrane proteins such as CD9 and CD63, exosome markers, and so on (Keerthikumar et al., 2016). There were 372 proteins common to the NSC exosomes and Exocarta (Bardou et al., 2014). Thus, our exosome samples shared many proteins with those previously reported (Figure 2A). The sample comparisons were "aExo vs. 


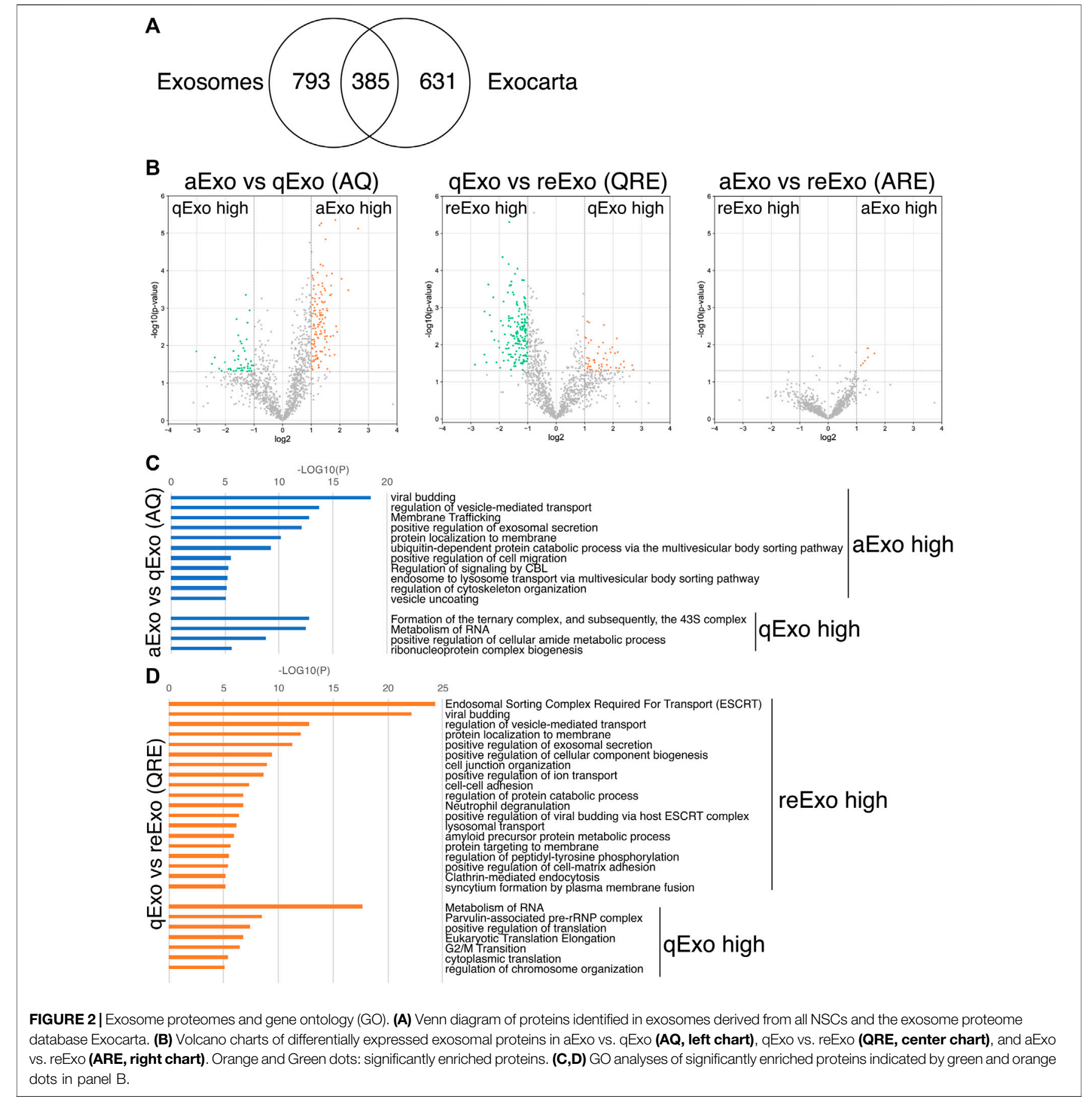

qExo" (AQ), "qExo vs. reExo" (QRE), and "aExo vs. reExo" (ARE) (Figures 2B,C). The volcano plots revealed bilateral significantly expressed proteins $\left(\log _{2}\right.$ fold change $(\mathrm{FC})>1$; $p$-value < 0.05) in AQ and QRE (Figure 2B; left and center charts). For ARE, however, there were only eight types of highly enriched proteins in aExo and no upregulated proteins in reExo relative to aExo (orange dots in right chart of Figure 2B; Supplementary Table S2).

To obtain functional insights of the differentially expressed protein cargos, we used gene lists of each comparison groups $\left(\log _{2}\right.$
FC > 1; p-value < 0.05) in a gene ontology (GO) analysis (Supplementary Table S2) conducted on the Metascape website (https://metascape.org/gp/index.html\#/main/step1). We identified enriched GO/KEGG and Reactome terms (Zhou et al., 2019). For both AQ and QRE, vesicle trafficking-related terms were significant in aExo, and translation-related terms were significant in qExo (Figure 2C; Supplementary Table S3). No GO analysis was conducted on ARE as there were too few enriched factors, and the functional clusters could not be calculated. We also applied the "molecular complex detection" 


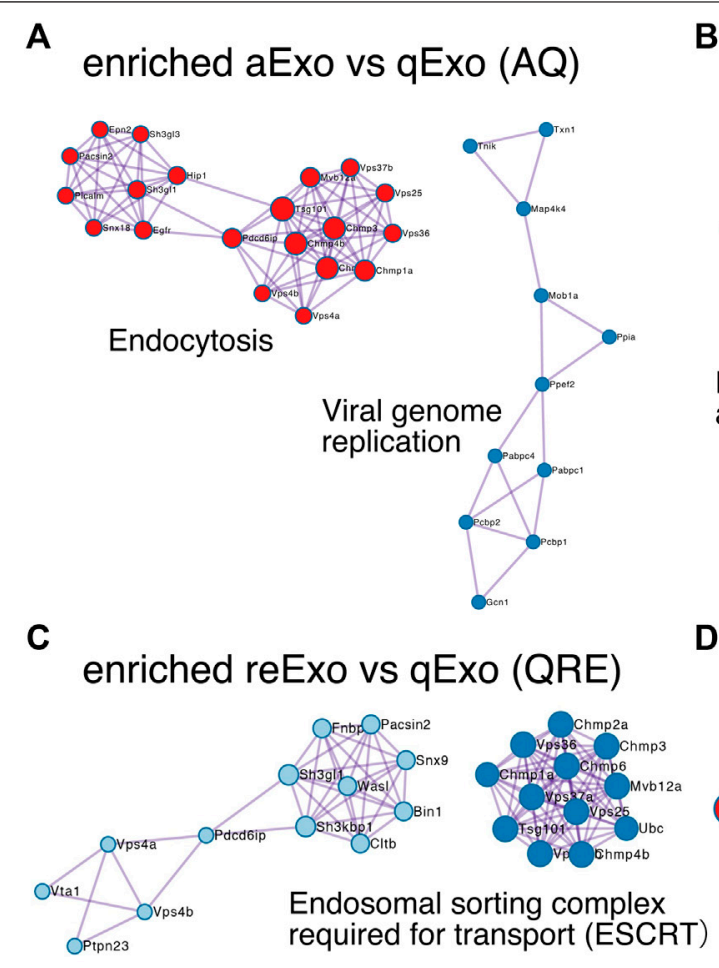

Membrane Trafficking

\section{enriched qExo vs aExo (AQ)}
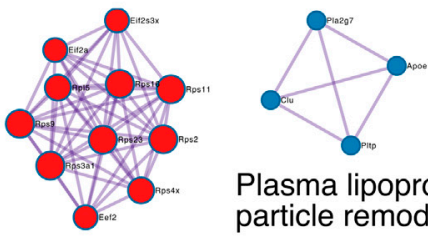

Plasma lipoprotein particle remodeling

Formation of the ternary complex, and subsequently, the $43 \mathrm{~S}$ complex

FIGURE 3 | Molecular complex detection networks among enriched proteins. Molecular complex detection networks of putative protein-protein interactions among enriched proteins in exosomes from aNSCs (aExo), exosomes from qNSCs (qExo), and exosomes from reNSCs (reExo). Each node color indicates a different network cluster. (A) Annotations enriched in aExo in aExo vs. qExo (AQ). (B) Annotations enriched in qExo in AQ. (C) Annotations enriched in reExo in qExo vs. reExo (QRE). (D) Annotations enriched in qExo in QRE.

(MCODE) algorithm to detect densely related regions in the pool of protein-protein interaction webs (Bader and Hogue, 2003) and explore potential connections among our exosome proteomes. The algorithm speculated high-confidence networks of highly enriched proteins in AQ (Figures 3A,B) and QRE (Figures 3C,D) and applied the enriched terms to annotate each MCODE network (Supplementary Tables S4). MCODE cluster identified endocytosis- and membrane traffickingrelated clusters as aExo- and reExo-enriched terms and translation- and ribosome-related clusters as the qExoenriched terms in both AQ and QRE comparisons. It was consistent with the elevated scores of ribosomal protein subunits in qExo proteome (Supplementary Table S4). The foregoing results disclosed the contrasts between the quiescence and proliferation stages and facilitated the exploration of potential functional units from exosome proteomes.

\section{Exosomes Participate in Quiescence Regulation of NSCs}

Our results demonstrated distinct differences in exosome protein contents and enriched pathways between the quiescence and proliferation stages of NSCs. We speculated that the high enrichment of translation-related proteins in qExo may imply the contribution of exosomes to reduce protein translation in qNSCs by discarding the contents from cells. To explore the role of exosomes in this hypothesis, we applied a chemical inhibitor of the exosome (Figure 4A). We used GW4869, a neutral, noncompetitive inhibitor of sphingomyelinase (N-SMase) which reduces ceramide levels and inhibits the ceramidemediated formation of mature exosomes in MVBs (Verderio et al., 2018). GW4869 is reported to inhibit exosome secretion by HEK293 cells, macrophages, and mesenchymal stem cells (Kosaka et al., 2010; Essandoh et al., 2015; Che et al., 2019). Nanosight measurements revealed that approximately one third of the exosome secretion was blocked in NSCs by GW4869 (Figure 4B). We selected GW4869 for our subsequent analyses and investigated its effects.

We next investigated whether reduced exosome secretion affects the induction of NSC quiescence in proliferating NSCs and the maintenance of quiescence in qNSCs. We tested CycD1 expression levels by WB analysis for the NSC proliferation criteria (Baldin et al., 1993). We treated NSCs with GW4869 and DMSO, as a negative control, dissolved in the quiescent medium. Contrasting CycD1 expression was detected $12 \mathrm{~h}$ after inducing quiescence with the exosome inhibitor. The cells treated with GW4869 displayed higher CycD1 expression 

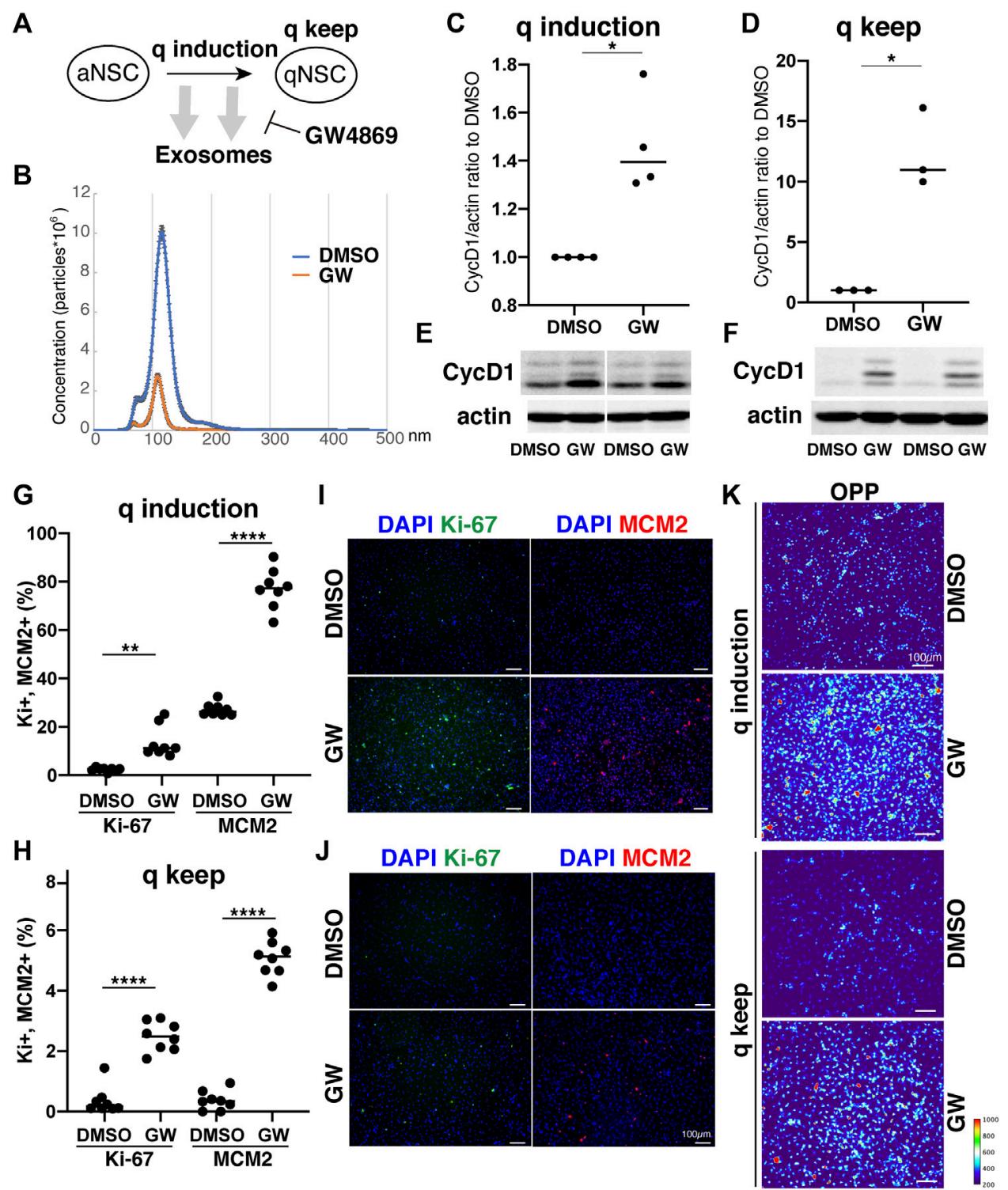

FIGURE 4 | Exosomes involved in cell cycle regulation of qNSCs. (A) Exosomal functions in quiescence entrance and maintenance. (B) Exosome quantification of aNSCs after treatment with GW4869 (orange) and DMSO as a control (blue). Horizontal axis: vesicle size. Vertical axis: vesicle number. (C,D) Quantification of CycD1 levels in NSCs after inducing (C) and maintaining quiescence (D) in presence of GW4869 for $12 \mathrm{~h}$ (C) and $24 \mathrm{~h}$ (D). All values were divided by that for DMSO control. Each plot indicates independent experiments. CycD1 band intensities were divided by those of actin bands. Ratios with respect to DMSO control are plotted. Middle lines in plots are medians. $\left({ }^{*} p<0.05,{ }^{* *} p<0.01\right.$; two-tailed paired t-test, $n=4$; For q-keep, one outlier identified by outlier test of Grubbs was removed). (E,F) Representative results of WB of quiescence induction (E) and maintenance (F). CycD1 were three bands, and actin is loading control. (G,H) Cell counts of Ki-67- and MCM2-positive cells after inducing (G) and maintaining quiescence (H) after incubation with GW4869 for 24 h. Ki-67-and MCM2-positive cell numbers were divided by the cell number based on DAPI staining and shown as the percentage. $\left({ }^{\star *} p=0.013,{ }^{* * * *} p<0.0001\right.$; one-way ANOVA, Turkey's multiple comparison test, $\left.n=8\right)$. (I,J) Representative photos of Ki-67- (green) and MCM2-positive cells (red) with DAPI staining (blue) in panel G (I) and $\mathrm{H}$ (J). (K) Protein synthesis activity in the quiescenceinduced ( $q$ induction) and maintained cells (q keep). The intensity of OP-puro labeling (OPP) is represented by a thermal scale.

levels than those in the DMSO control (Figures 4C,E). Hence, interference of exosome biogenesis and secretion impeded the NSCs from entering the G0 phase. We treated qNSCs with GW4869 in the quiescent medium to certify the influence of hindered exosome activity on the maintenance of quiescence. We discovered that after $24 \mathrm{~h}, \mathrm{CycD} 1$ expression was upregulated in the presence of GW4869 (Figures 4D,F). We investigated two proliferation markers, Ki-67 and MCM2, which are widely used to detect proliferating NSCs, in GW4869-treated NSCs. MCM2 is highly expressed throughout the cell cycle, including the G1 phase and except the G0 phase, whereas Ki-67 expression peaks at M phases and is low at the G1 phase (Miller et al., 2018; Harris et al., 
2021). Exosome inhibition increased the percentages of proliferating (Ki-67+ and MCM2+) cells in both quiescentinduced and quiescent-mainteined NSCs (Figures 4G-J). Thus, the blockage of exosome secretion delayed the entry of proliferating NSCs into the G0 phases and destablized the G0 phase.

To elucidate the mechanism through which exosomal inhibition destabilized the G0 phase in NSC quiescence, protein translation was measured using a puromycin analogue, OP-puro (OPP). OPP is effectively incorporated into newly generated proteins and can be used to quantify the protein synthesis rates in cells by detecting OPP-labeled proteins after fixation (Liu et al., 2012). aNSCs and qNSCs were incubated in GW-containing quiescent medium for $24 \mathrm{~h}$, labeled with OPP for $1 \mathrm{~h}$, and analyzed. Exosome inhibition increased the levels of OPP-labeled proteins in quiescent-induced and quiescent-maintained NSCs compared to those in the controls (Figure 4K). This higher translation in GW-treated NSCs was consistent with higher percentages of proliferating cells in those than in controls under quiescent culture conditions (Figures 4G-J). Taken together, these results demonstrated a strong correlation between exosome secretion and cell cycle regulation together with protein synthesis in NSCs quiescence.

\section{DISCUSSION}

Exosomes are ubiquitously secreted by nearly all cell types and thought to actively participate in various niche signal transductions (Thery et al., 2009; Rashed et al., 2017; Bonafina et al., 2020). In this study, we successfully purified exosomes from NSCs that were available for proteomics (Figure 1). The exosomal proteomes we acquired contained not only many common proteins with those previously reported but also uncommon proteins that may specifically exist in NSCs (Figure 2). Moreover, our results highlighted the different cargo in exosomes from the same NSC origin but at different states (Figure 3). Exosomes are known as biomarkers of cancers to inform the origins and biological changes. Our MS data might contribute to specify the condition and maintenance of NSC activation and quiescence in the adult brain in future.

We initially tested several exosome collection kits and methods but discovered that they introduced protein contamination. Ultracentrifugation provided high-purity exosomes but the yield was too low due to sample loss during the procedure (Figure 1). Hence, we increased the cell number, culture medium volume, and recovering time after ultracentrifuge and finally obtained high-purity, high-yield exosomes. We then conducted TMT-based quantitative proteomics by nanoLC/MS/ MS analyses to clarify the roles of exosomes from NSCs. A GO analysis of the enriched proteins at each state disclosed functional clusters that facilitated determination of the roles of exosomes (Figures 2, 3). It was established that vesicular transport and protein translation were enriched pathways in the proliferating and quiescent exosomes, respectively.
A prevalence of ribosomal proteins has been reported for $\mathrm{sEVs}$ from cancer cells (Mathivanan et al., 2010; Ji et al., 2013; Willms et al., 2016). Our study showed that there was apparent concentrated expression of $40 \mathrm{~S}$ ribosomal subunit, which forms a pre-initiation complex for translation (Jackson et al., 2010) in qExo. As quiescent cells have low protein synthesis rates, exosomes may export translation-related components from cells during quiescence induction. The "discarding model" of exosome function upon entering quiescence may explain the fact that ribosomal protein expression levels rise in exosomes but fall in cells (Pan et al., 1985; Mukherjee et al., 2016). However, the discarding model does not rule out the possibility that the exosome cargos may transfer signals to recipient cells or alter their microenvironments, for example by transferring ribosomes from quiescent cells to other cells. Future research should explore the molecular function of specific proteins within our exosome proteomes as some of them might be essential for tuning cell proliferation from quiescence. For example, PCSK6 (also called PACE4) (Supplementary Table S2), enriched in aExo, is a protein convertase that could promote cell proliferation by inducing the ERK1/2 and STAT3 signaling pathways (Seidah et al., 2013; Jiang et al., 2017). It would be an attractive target if the specific factor or exosomes may reactivate NSCs in the adult brain in order to avoid the functional decline of brain with aging and brain disorders.

Finally, our study revealed that exosome production and secretion regulate NSC quiescence using the exosome inhibitor GW4869 (Figure 4). First, we found that the exosome inhibitor increased CycD1 levels in qNSCs, which indicated the re-entry of qNSCs to the cell cycle, and prevented the reduction in CycD1 in aNSCs during the entrance to the G0 phase after quiescence induction. Second, the exosome inhibitor significantly increased the number of proliferating cells expressing Ki-67 and MCM2, even under quiescent culture conditions. Third, protein translation was greatly upregulated in qNSCs and quiescenceinduced NSCs. These results suggested that interference with exosome biogenesis and secretion impedes the maintenance of and transition to quiescence in NSCs by upregulating translation. The "discarding model" we described previously herein might be appropriate based on the results indicating that exosomes exhaust the functional translational machinery to reduce new protein synthesis for proliferation. Further research will be required to confirm this hypothesis and to identify the molecular mechanisms connecting proliferation with protein synthesis.

To analyze exosome function, we used GW4869, the most widely used inhibitor of exosome production, to prevent exosome secretion. This inhibitor has other functions such as interrupting sphingolipid metabolism and ceramide production. Proliferating aNSCs did not alter cycD1 levels and proliferation markers (Ki-67 expression and DNA analogue uptake) after treated with GW4869 (not shown). Based on these results, it is likely that the sphingolipid metabolism alteration does not have a direct effect on the proliferation of NSCs.

A recent report demonstrated that proliferating NSCs gradually enter shallow quiescence with age and transfer between two states, quiescence and proliferation, and the balance is regulated by the degradation of a specific 
transcriptional factor, Ascl1 (Harris et al., 2021). Exosomal regulation of new protein synthesis, we hypothesized here, is potentially a novel mechanism regulating this balance in the adult brain. Taken together, our results established the functional importance of exosomes in participating in the quiescence regulation of NSC quiescence.

\section{DATA AVAILABILITY STATEMENT}

The raw data and analysis files have been deposited to the ProteomeXchange Consortium (http://proteomecentral. proteomexchange.org) via the jPOST partner repository (https://jpostdb.org) under the data set identifier PXD027651.

\section{AUTHOR CONTRIBUTIONS}

JZ and TK designed and conducted all experiments. JU, KI, and YI performed the proteomic analyses. JZ, TK, KI, YI, and RK wrote the manuscript.

\section{FUNDING}

This work was supported by a Grant-in-Aid for Scientific Research (B) (JSPS No. 20H03260) (to TK), the Japan Agency

\section{REFERENCES}

Bader, G. D., and Hogue, C. W. (2003). An Automated Method for Finding Molecular Complexes in Large Protein Interaction Networks. BMC Bioinformatics 4, 2. doi:10.1186/1471-2105-4-2

Baldin, V., Lukas, J., Marcote, M. J., Pagano, M., and Draetta, G. (1993). Cyclin D1 Is a Nuclear Protein Required for Cell Cycle Progression in G1. Genes Develop. 7, 812-821. doi:10.1101/gad.7.5.812

Bardou, P., Mariette, J., Escudié, F., Djemiel, C., and Klopp, C. (2014). Jvenn: an Interactive Venn Diagram Viewer. BMC Bioinformatics 15, 293. doi:10.1186/ 1471-2105-15-293

Bátiz, L. F., Castro, M. A., Burgos, P. V., Velásquez, Z. D., Muñoz, R. I., Lafourcade, C. A., et al. (2015). Exosomes as Novel Regulators of Adult Neurogenic Niches. Front Cel Neurosci 9, 501. doi:10.3389/fncel.2015.00501

Bonafina, A., Paratcha, G., and Ledda, F. (2020). Deciphering New Players in the Neurogenic Adult Hippocampal Niche. Front. Cel Dev. Biol. 8, 548. doi:10.3389/ fcell.2020.00548

Cavallucci, V., Fidaleo, M., and Pani, G. (2016). Neural Stem Cells and Nutrients: Poised between Quiescence and Exhaustion. Trends Endocrinol. Metab. 27, 756-769. doi:10.1016/j.tem.2016.06.007

Che, Y., Shi, X., Shi, Y., Jiang, X., Ai, Q., Shi, Y., et al. (2019). Exosomes Derived from miR-143-Overexpressing MSCs Inhibit Cell Migration and Invasion in Human Prostate Cancer by Downregulating TFF3. Mol. Ther. - Nucleic Acids 18, 232-244. doi:10.1016/j.omtn.2019.08.010

Cheung, T. H., and Rando, T. A. (2013). Molecular Regulation of Stem Cell Quiescence. Nat. Rev. Mol. Cel Biol. 14, 329-340. doi:10.1038/nrm3591

Codega, P., Silva-Vargas, V., Paul, A., Maldonado-Soto, A. R., Deleo, A. M., Pastrana, E., et al. (2014). Prospective Identification and Purification of Quiescent Adult Neural Stem Cells from Their In Vivo Niche. Neuron 82, 545-559. doi:10.1016/j.neuron.2014.02.039

Colombo, M., Raposo, G., and Théry, C. (2014). Biogenesis, Secretion, and Intercellular Interactions of Exosomes and Other Extracellular Vesicles. for Medical Research and Development (AMED) under Grant Nos. JP20gm6410006 (to TK) and JP19gm1110002 (to RK), and SPIRITS 2021 of Kyoto University (to TK), and by JST PRESTO (No. JPMJPR18H2) (to KI).

\section{ACKNOWLEDGMENTS}

The authors thank P. Kurre, J. Hejna, Y. Okuno, H. Kouda, K. Furuta-Okamoto, K. Ishii and members of the Kageyama Laboratory for their technical assistance and discussions.

\section{SUPPLEMENTARY MATERIAL}

The Supplementary Material for this article can be found online at: https:/www.frontiersin.org/articles/10.3389/fcell.2021.762293/ full\#supplementary-material

\section{Supplementary Methods | Methods for mass spectrometory.}

Supplementary Table S1 | Proteins identified using proteomic analysis.

Supplementary Table S2 | Differential expression of proteins in each exosome sample. Intensity 1-3 represents triplicate samples of exosomes from aNSCs (aExo samples). Intensity 4-6 represents triplicate samples of exosomes from qNSCs (qExo samples). Intensity 7-9 represents triplicate samples of exosomes from reNSCs (reExo samples).

Supplementary Table S3 | Gene ontology analysis of enriched proteomes.

Supplementary Table S4 | Gene ontology annotation lists.

Annu. Rev. Cel Dev. Biol. 30, 255-289. doi:10.1146/annurev-cellbio-101512122326

Cossetti, C., Iraci, N., Mercer, T. R., Leonardi, T., Alpi, E., Drago, D., et al. (2014). Extracellular Vesicles from Neural Stem Cells Transfer IFN- $\gamma$ via Ifngr1 to Activate Stat1 Signaling in Target Cells. Mol. Cel 56, 193-204. doi:10.1016/ j.molcel.2014.08.020

Delgado, A. C., Ferrón, S. R., Vicente, D., Porlan, E., Perez-Villalba, A., Trujillo, C. M., et al. (2014). Endothelial NT-3 Delivered by Vasculature and CSF Promotes Quiescence of Subependymal Neural Stem Cells through Nitric Oxide Induction. Neuron 83, 572-585. doi:10.1016/j.neuron.2014.06.015

Dolo, V., Ginestra, A., Cassarà, D., Violini, S., Lucania, G., Torrisi, M. R., et al. (1998). Selective Localization of Matrix Metalloproteinase 9, Beta1 Integrins, and Human Lymphocyte Antigen Class I Molecules on Membrane Vesicles Shed by 8701-BC Breast Carcinoma Cells. Cancer Res. 58, 4468-4474.

Essandoh, K., Yang, L., Wang, X., Huang, W., Qin, D., Hao, J., et al. (2015). Blockade of Exosome Generation with GW4869 Dampens the Sepsis-Induced Inflammation and Cardiac Dysfunction. Biochim. Biophys. Acta (Bba) - Mol. Basis Dis. 1852, 2362-2371. doi:10.1016/j.bbadis.2015.08.010

Fuentealba, L. C., Obernier, K., and Alvarez-Buylla, A. (2012). Adult Neural Stem Cells Bridge Their Niche. Cell Stem Cell 10, 698-708. doi:10.1016/ j.stem.2012.05.012

Harris, L., Rigo, P., Stiehl, T., Gaber, Z. B., Austin, S. H. L., Masdeu, M. d. M., et al. (2021). Coordinated Changes in Cellular Behavior Ensure the Lifelong Maintenance of the Hippocampal Stem Cell Population. Cell Stem Cell 28, 863-876. e866. doi:10.1016/j.stem.2021.01.003

Hoshino, A., Costa-Silva, B., Shen, T.-L., Rodrigues, G., Hashimoto, A., Tesic Mark, M., et al. (2015). Tumour Exosome Integrins Determine Organotropic Metastasis. Nature 527, 329-335. doi:10.1038/nature15756

Huang, J., Ding, Z., Luo, Q., and Xu, W. (2019). Cancer Cell-Derived Exosomes Promote Cell Proliferation and Inhibit Cell Apoptosis of Both normal Lung Fibroblasts and Non-Small Cell Lung Cancer Cell through Delivering AlphaSmooth Muscle Actin. Am. J. Transl Res. 11, 1711-1723. 
Jackson, R. J., Hellen, C. U. T., and Pestova, T. V. (2010). The Mechanism of Eukaryotic Translation Initiation and Principles of its Regulation. Nat. Rev. Mol. Cel Biol. 11, 113-127. doi:10.1038/nrm2838

Ji, H., Greening, D. W., Barnes, T. W., Lim, J. W., Tauro, B. J., Rai, A., et al. (2013). Proteome Profiling of Exosomes Derived from Human Primary and Metastatic Colorectal Cancer Cells Reveal Differential Expression of Key Metastatic Factors and Signal Transduction Components. Proteomics 13, 1672-1686. doi:10.1002/pmic.201200562

Jiang, H., Wang, L., Wang, F., and Pan, J. (2017). Proprotein Convertase Subtilisin/ kexin Type 6 Promotes In Vitro Proliferation, Migration and Inflammatory Cytokine Secretion of Synovial Fibroblast-Like Cells from Rheumatoid Arthritis via Nuclear-Kb, Signal Transducer and Activator of Transcription 3 and Extracellular Signal Regulated 1/2 Pathways. Mol. Med. Rep. 16, 8477-8484. doi:10.3892/mmr.2017.7595

Keerthikumar, S., Chisanga, D., Ariyaratne, D., Al Saffar, H., Anand, S., Zhao, K., et al. (2016). ExoCarta: A Web-Based Compendium of Exosomal Cargo. J. Mol. Biol. 428, 688-692. doi:10.1016/j.jmb.2015.09.019

Kjell, J., Fischer-Sternjak, J., Thompson, A. J., Friess, C., Sticco, M. J., Salinas, F., et al. (2020). Defining the Adult Neural Stem Cell Niche Proteome Identifies Key Regulators of Adult Neurogenesis. Cell Stem Cell 26, 277-293. doi:10.1016/ j.stem.2020.01.002

Kobayashi, T., and Kageyama, R. (2021). Lysosomes and Signaling Pathways for Maintenance of Quiescence in Adult Neural Stem Cells. FEBS J. 288, 3082-3093. doi:10.1111/febs.15555

Kobayashi, T., Piao, W., Takamura, T., Kori, H., Miyachi, H., Kitano, S., et al. (2019). Enhanced Lysosomal Degradation Maintains the Quiescent State of Neural Stem Cells. Nat. Commun. 10, 5446. doi:10.1038/s41467-019-13203-4

Kosaka, N., Iguchi, H., Yoshioka, Y., Takeshita, F., Matsuki, Y., and Ochiya, T. (2010). Secretory Mechanisms and Intercellular Transfer of microRNAs in Living Cells. J. Biol. Chem. 285, 17442-17452. doi:10.1074/jbc.m110.107821

Lepko, T., Pusch, M., Müller, T., Schulte, D., Ehses, J., Kiebler, M., et al. (2019). Choroid Plexus-Derived miR-204 Regulates the Number of Quiescent Neural Stem Cells in the Adult Brain. EMBO J. 38, e100481. doi:10.15252/embj.2018100481

Liu, J., Xu, Y., Stoleru, D., and Salic, A. (2012). Imaging Protein Synthesis in Cells and Tissues with an Alkyne Analog of Puromycin. Proc. Natl. Acad. Sci. 109, 413-418. doi:10.1073/pnas.1111561108

Martynoga, B., Mateo, J. L., Zhou, B., Andersen, J., Achimastou, A., Urban, N., et al. (2013). Epigenomic Enhancer Annotation Reveals a Key Role for NFIX in Neural Stem Cell Quiescence. Genes Develop. 27, 1769-1786. doi:10.1101/gad.216804.113

Mathieu, M., Martin-Jaular, L., Lavieu, G., and Théry, C. (2019). Specificities of Secretion and Uptake of Exosomes and Other Extracellular Vesicles for Cell-To-Cell Communication. Nat. Cel Biol. 21, 9-17. doi:10.1038/s41556-018-0250-9

Mathivanan, S., Lim, J. W. E., Tauro, B. J., Ji, H., Moritz, R. L., and Simpson, R. J. (2010). Proteomics Analysis of A33 Immunoaffinity-Purified Exosomes Released from the Human colon Tumor Cell Line LIM1215 Reveals a Tissue-Specific Protein Signature. Mol. Cell Proteomics 9, 197-208. doi:10.1074/mcp.m900152-mcp200

Matsumoto, Y., Kano, M., Murakami, K., Toyozumi, T., Suito, H., Takahashi, M., et al. (2020). Tumor-Derived Exosomes Influence the Cell Cycle and Cell Migration of Human Esophageal Cancer Cell Lines. Cancer Sci. 111, 4348-4358. doi:10.1111/cas.14660

Miller, I., Min, M., Yang, C., Tian, C., Gookin, S., Carter, D., et al. (2018). Ki67 Is a Graded Rather Than a Binary Marker of Proliferation versus Quiescence. Cel Rep. 24, 1105-1112. doi:10.1016/j.celrep.2018.06.110

Moriya, Y., Kawano, S., Okuda, S., Watanabe, Y., Matsumoto, M., Takami, T., et al. (2019). The jPOST Environment: an Integrated Proteomics Data Repository and Database. Nucleic Acids Res. 47, D1218-D1224. doi:10.1093/nar/gky899

Morton, M. C., Neckles, V. N., Seluzicki, C. M., Holmberg, J. C., and Feliciano, D. M. (2018). Neonatal Subventricular Zone Neural Stem Cells Release Extracellular Vesicles that Act as a Microglial Morphogen. Cel Rep. 23, 78-89. doi:10.1016/j.celrep.2018.03.037

Mukherjee, S., Brulet, R., Zhang, L., and Hsieh, J. (2016). REST Regulation of Gene Networks in Adult Neural Stem Cells. Nat. Commun. 7, 13360. doi:10.1038/ ncomms 13360
Ogata, K., and Ishihama, Y. (2020). Extending the Separation Space with Trapped Ion Mobility Spectrometry Improves the Accuracy of Isobaric Tag-Based Quantitation in Proteomic LC/MS/MS. Anal. Chem. 92, 8037-8040. doi:10.1021/acs.analchem.0c01695

Pan, B. T., Teng, K., Wu, C., Adam, M., and Johnstone, R. M. (1985). Electron Microscopic Evidence for Externalization of the Transferrin Receptor in Vesicular Form in Sheep Reticulocytes. J. Cel Biol. 101, 942-948. doi:10.1083/jcb.101.3.942

Parsons, M. E. M., Mcparland, D., Szklanna, P. B., Guang, M. H. Z., O'Connell, K., O'Connor, H. D., et al. (2017). A Protocol for Improved Precision and Increased Confidence in Nanoparticle Tracking Analysis Concentration Measurements between 50 and $120 \mathrm{Nm}$ in Biological Fluids. Front. Cardiovasc. Med. 4, 68. doi:10.3389/fcvm.2017.00068

Rashed, M. H., Bayraktar, E., Gouda, K. H., Abd-Ellah, M. F., Amero, P., ChavezReyes, A., et al. (2017). Exosomes: From Garbage Bins to Promising Therapeutic Targets. Int. J. Mol. Sci. 18, 538. doi:10.3390/ijms18030538

Seidah, N. G., Sadr, M. S., Chrétien, M., and Mbikay, M. (2013). The Multifaceted Proprotein Convertases: Their Unique, Redundant, Complementary, and Opposite Functions. J. Biol. Chem. 288, 21473-21481. doi:10.1074/jbc.r113.481549

Stronati, E., Conti, R., Cacci, E., Cardarelli, S., Biagioni, S., and Poiana, G. (2019). Extracellular Vesicle-Induced Differentiation of Neural Stem Progenitor Cells. Int. J. Mol. Sci. 20, 3691. doi:10.3390/ijms20153691

Théry, C., Amigorena, S., Raposo, G., and Clayton, A. (2006). Isolation and Characterization of Exosomes from Cell Culture Supernatants and Biological Fluids. Curr. Protoc. Cel Biol. 3, 22. doi:10.1002/0471143030.cb0322s30

Théry, C., Ostrowski, M., and Segura, E. (2009). Membrane Vesicles as Conveyors of Immune Responses. Nat. Rev. Immunol. 9, 581-593. doi:10.1038/nri2567

Urbán, N., Blomfield, I. M., and Guillemot, F. (2019). Quiescence of Adult Mammalian Neural Stem Cells: A Highly Regulated Rest. Neuron 104, 834-848. doi:10.1016/j.neuron.2019.09.026

Valadi, H., Ekström, K., Bossios, A., Sjöstrand, M., Lee, J. J., and Lötvall, J. O. (2007). Exosome-Mediated Transfer of mRNAs and microRNAs Is a Novel Mechanism of Genetic Exchange between Cells. Nat. Cel Biol. 9, 654-659. doi:10.1038/ncb1596

Van Niel, G., D'angelo, G., and Raposo, G. (2018). Shedding Light on the Cell Biology of Extracellular Vesicles. Nat. Rev. Mol. Cel Biol. 19, 213-228. doi:10.1038/nrm.2017.125

Verderio, C., Gabrielli, M., and Giussani, P. (2018). Role of Sphingolipids in the Biogenesis and Biological Activity of Extracellular Vesicles. J. Lipid Res. 59, 1325-1340. doi:10.1194/jlr.r083915

Willms, E., Johansson, H. J., Mäger, I., Lee, Y., Blomberg, K. E. M., Sadik, M., et al. (2016). Cells Release Subpopulations of Exosomes with Distinct Molecular and Biological Properties. Sci. Rep. 6, 22519. doi:10.1038/srep22519

Zhou, Y., Zhou, B., Pache, L., Chang, M., Khodabakhshi, A. H., Tanaseichuk, O., et al. (2019). Metascape Provides a Biologist-Oriented Resource for the Analysis of Systems-Level Datasets. Nat. Commun. 10, 1523. doi:10.1038/s41467-019-09234-6

Conflict of Interest: The authors declare that the research was conducted in the absence of any commercial or financial relationships that could be construed as a potential conflict of interest.

Publisher's Note: All claims expressed in this article are solely those of the authors and do not necessarily represent those of their affiliated organizations, or those of the publisher, the editors and the reviewers. Any product that may be evaluated in this article, or claim that may be made by its manufacturer, is not guaranteed or endorsed by the publisher.

Copyright $\odot 2021$ Zhang, Uchiyama, Imami, Ishihama, Kageyama and Kobayashi. This is an open-access article distributed under the terms of the Creative Commons Attribution License (CC BY). The use, distribution or reproduction in other forums is permitted, provided the original author(s) and the copyright owner(s) are credited and that the original publication in this journal is cited, in accordance with accepted academic practice. No use, distribution or reproduction is permitted which does not comply with these terms. 


\section{GLOSSORY}

aExo: aNSC exosomes

aNSCs: active neural stem cells

AQ: aExo vs. qExo

ARE: aExo vs. reExo

BMP: bone morphogenic protein

CSF: cerebrospinal fluid

DMSO: dimethyl sulfoxide

EGF: epidermal growth factor

ESCRT: endosomal sorting complexes for transport

GO: gene ontology

KEGG: Kyoto Encyclopedia of Genes and Genomes

HRP: horseradish peroxidase

ILVs: intraluminal vesicles

LC: liquid chromatography

MCODE: molecular complex detection

miRNAs: microRNAs

MS: mass spectrometry

NSPCs: neural stem/progenitor cells
NTA: nanoparticle tracking analysis

MVE: multiple vesicle endosome

nano LC/MS/MS: nanoscale liquid chromatography-tandem mass spectrometry

NSCs: neural stem cells

N-Smase: neutral-sphingomyelinase

OPP: O-propargyl-puromycin (OP-puro)

PGD2: prostaglandin-D2

qExo: qNSC exosomes

qNSCs: quiescent neural stem cells

QRE: qExo vs. reExo

reExo: reNSC exoxomes

reNSCs: reactivating neural stem cells

sEVs: small extracellular vesicles

S1P: sphingosine-1-phosphate

SDS-PAGE: sodium dodecyl sulfate polyacrylamide gel electrophoresis

TCA: trichloroacetic acid

TEM: transmission electron microscopy

TMT: tandem mass tag

WB: western blotting 